\title{
Editorial
}

\section{Electrokinetics in Earth Sciences}

\author{
Laurence Jouniaux, ${ }^{1}$ and Tsuneo Ishido ${ }^{2}$ \\ ${ }^{1}$ Institut de Physique du Globe de Strasbourg, CNRS UMR7516 and Université de Strasbourg, 67084 Strasbourg Cedex, France \\ ${ }^{2}$ Geological Survey of Japan, AIST, Central 7, Tsukuba 305-8567, Japan
}

Correspondence should be addressed to Laurence Jouniaux; laurence.jouniaux@unistra.fr

Received 29 November 2012; Accepted 29 November 2012

Copyright (c) 2013 L. Jouniaux and T. Ishido. This is an open access article distributed under the Creative Commons Attribution License, which permits unrestricted use, distribution, and reproduction in any medium, provided the original work is properly cited.

Electrokinetics is used in earth sciences to provide insights concerning near-surface earth structures (from a few hundred meters up to several thousand meters depth) that host interstitial fluids (water, ice, oil, and gas). Electrokinetic phenomena are induced by the relative motion between the fluid and the rock matrix and, therefore, can provide information about the fluids within the earth. Self-potentials have been measured in hydrology, in active volcanic areas, and in polluted environments. Various methods based on electrokinetics, including self-potentials and seismoelectromagnetic conversion, have been applied to the management of hydraulic and hydrocarbon reservoirs, the resource prospecting in glaciated regions, the exploration for and monitoring of geothermal reservoirs, the characterization of fractured reservoirs, and so forth.

Electrokinetic phenomena have been known for more than two centuries (Reuss, 1809). Electrokinetic potential has been studied in relation to the field of colloid science and also in petroleum engineering research through its effects upon SP logging. Instrumentation improvements have resulted in an increased number of field observations, giving rise to new theoretical insights, specifically Pride's theory (1994) which combines Biot's equations and Maxwell's equations, and the developments by Garambois and Dietrich (2001) on the transfer functions between the electromagnetic field and the seismic field. This also arose new interest in modeling studies and led to numerous other publications in these fields during the past few decades.

This special issue addresses both self-potential and seismoelectrical conversions. The themes include field observations, modeling at small- and large-scale experimental developments, and theoretical analysis. From thirteen submissions, eleven papers were selected for publication in the special issue. Two reviewers critiqued each paper, and the manuscripts were then revised appropriately. We also include a tutorial which presents the basic principles of the coupling between fluid and electrical flow under both steady and unsteady conditions as developed by Pride (1994), and a review of various applications such as geothermal reservoir characterization, as well as an explanation about the electric double layer, whose assumptions are discussed in the paper of M. D. Jackson and E. Leinov. Field observations of selfpotential caused by flow in fractured reservoirs are described by Y. Nishi and T. Ishido; observed SP on mountain slopes is examined by T. N. Goto et al.; the self-potential induced by horizontal fluid flow is modeled by G. A. Skianis.

Seismic wave propagation induces a relative movement between the fluid and the rock matrix, leading to electrokinetic effects. Electrical signals that might arise due to earthquakes are described by A. Takeuchi et al. The seismoelectric method could be extended in future decades using interferometry by cross-correlation, as developed by $\mathrm{F}$. C. Schoemaker et al.

To properly interpret observations of self-potential and seismoelectric conversion, we need a better quantitative understanding of the electrokinetic coupling. The effect of deformation on this coupling is measured in granite by $\mathrm{O}$. Kuwano and S. Yoshida. The frequency dependence of this coupling is reviewed by L. Jouniaux and C. Bordes, and new laboratory experiments are described by P. W. J. Glover et al. (part 1 and part 2).

Laurence Jouniaux Tsuneo Ishido 

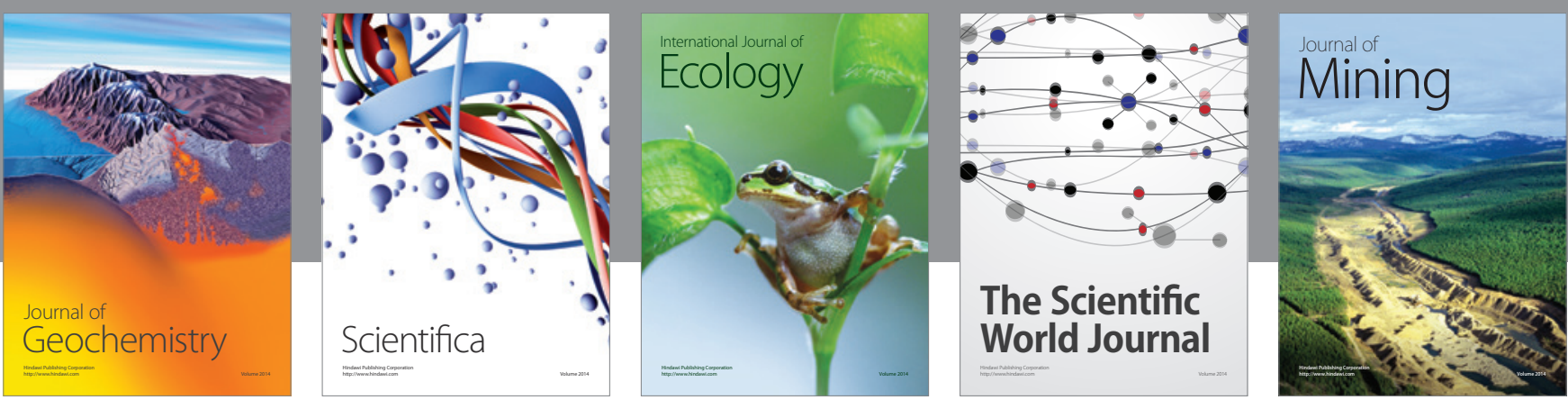

The Scientific World Journal
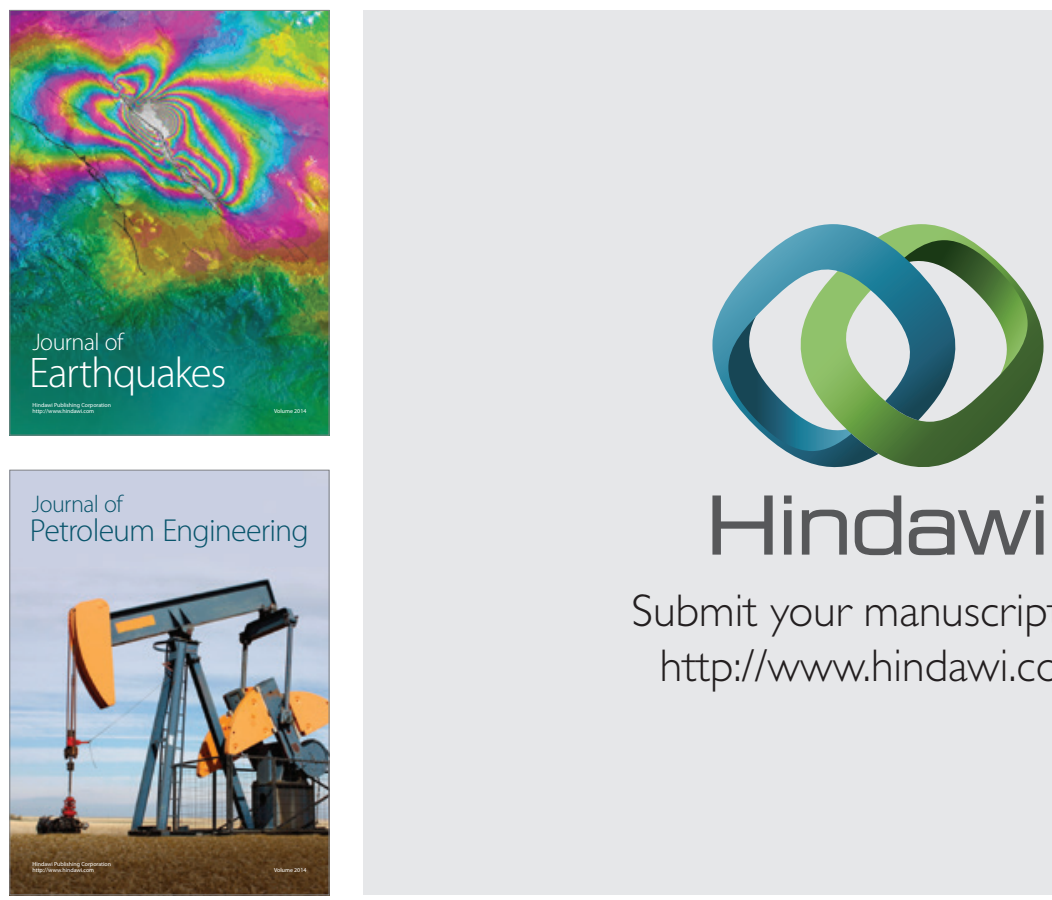

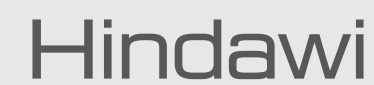

Submit your manuscripts at

http://www.hindawi.com
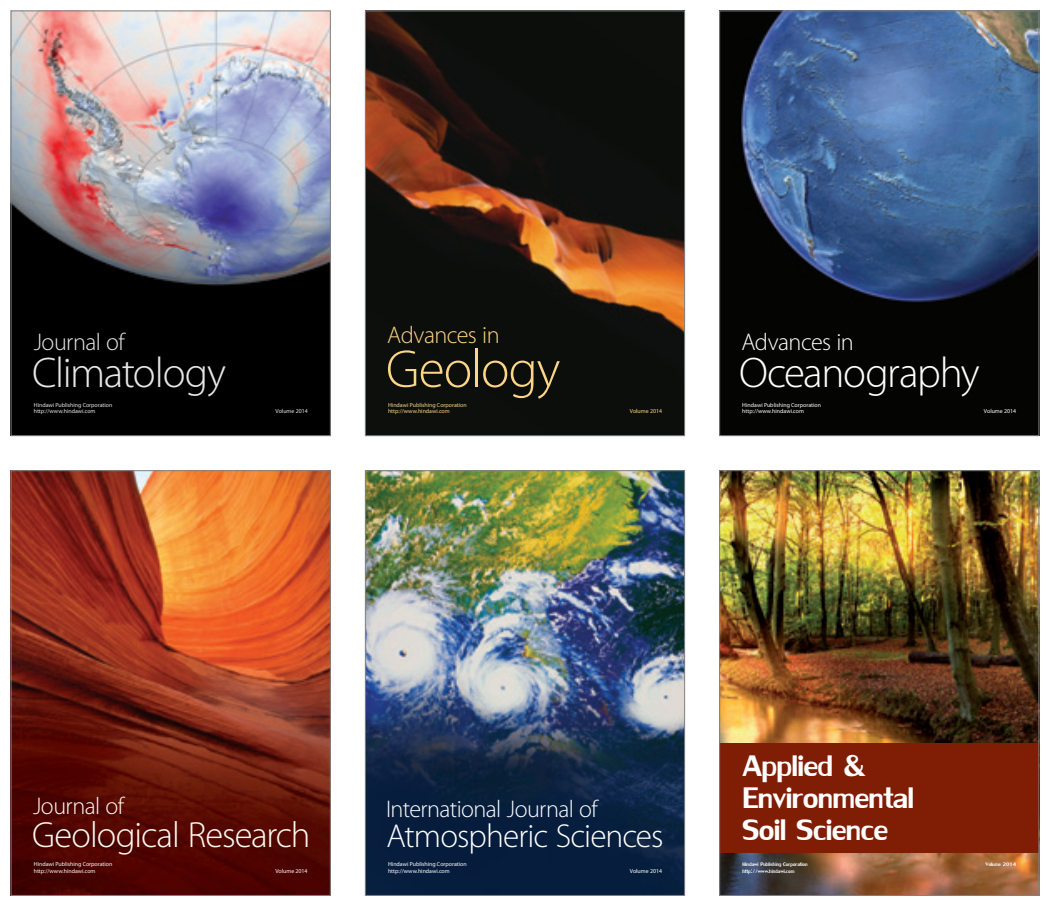
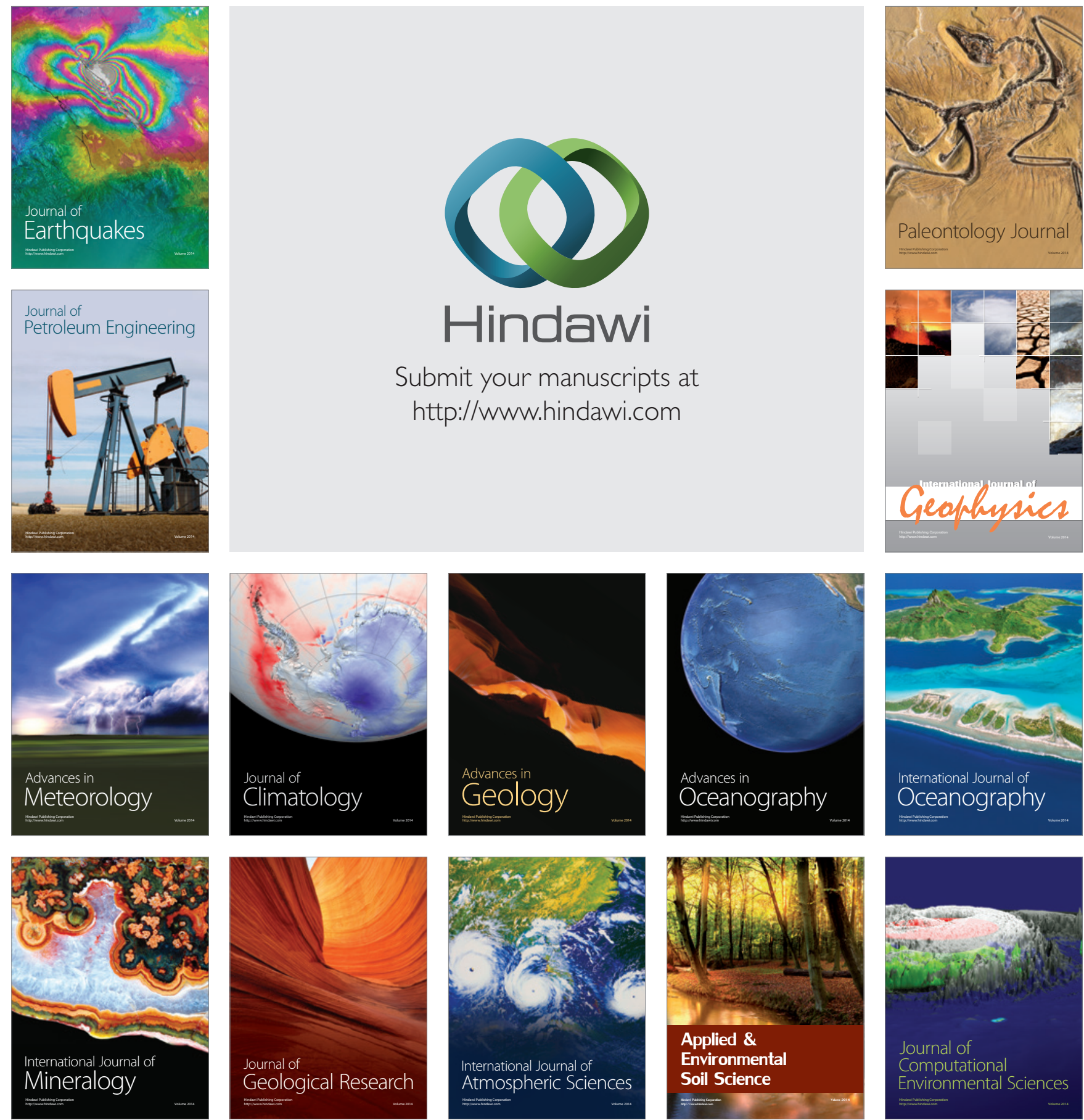